\title{
Ram pressure stripping of tilted galaxies
}

\author{
P. Jáchym ${ }^{1}$, J. Köppen ${ }^{1,2,3,4}$, J. Palouš ${ }^{1}$, and F. Combes ${ }^{5}$ \\ 1 Astronomical Institute, Academy of Sciences of the Czech Republic, Boční II 1401, 14131 Prague 4, Czech Republic \\ e-mail: jachym@ig.cas.cz, palous@ig.cas.cz \\ 2 Observatoire Astronomique de Strasbourg, 11 Rue de l'Université, 67000 Strasbourg, France \\ e-mail: koppen@astro.u-strasbg.fr \\ 3 International Space University, Parc d'Innovation, 1 Rue Jean-Dominique Cassini, 67400 Illkirch-Graffenstaden, France \\ ${ }^{4}$ Institut für Theoretische Physik und Astrophysik, Universität Kiel, 24098 Kiel, Germany \\ 5 Observatoire de Paris, LERMA, 61 Av. de l'Observatoire, 75014 Paris, France \\ e-mail: francoise.combes@obspm.fr
}

Received 4 December 2008 / Accepted 30 March 2009

\begin{abstract}
Context. Ram pressure stripping of galaxies in clusters can yield gas deficient disks. Previous numerical simulations based on various approaches suggested that, except for near edge-on disk orientations, the amount of stripping depends very little on the inclination angle.

Aims. Following our previous numerical and analytical study of face-on stripping, we extend the set of parameters with the disk tilt angle and explore in detail the effects of the ram pressure on the interstellar content (ISM) of tilted galaxies that orbit in various environments of clusters, with compact or extended distributions of the intra-cluster medium (ICM). We further study how results of numerical simulations could be estimated analytically. To isolate the effect of inclination, galaxies on strictly radial orbits are considered.

Methods. A grid of numerical simulations with varying parameters is produced using the tree/SPH code GADGET with a modified method for calculating the ISM-ICM interaction. These SPH calculations extend the set of existing results obtained from different codes using various numerical techniques.

Results. The simulations confirm the general trend of less stripping at orientations close to edge-on. The dependence on the disk tilt angle is more pronounced for compact ICM distributions, however it almost vanishes for strong ram pressure pulses. Although various hydrodynamical effects are present in the ISM-ICM interaction, the main quantitative stripping results appear to be roughly consistent with a simple scenario of momentum transfer from the encountered ICM. This behavior can also be found in previous simulations. To reproduce the numerical results we propose a fitting formula depending on the disk tilt angle and on the column density of the encountered ICM. Such a dependence is superior to that on the peak ram pressure used in previous simple estimates.
\end{abstract}

Key words. galaxies: clusters: general - galaxies: intergalactic medium - galaxies: general - ISM: structure - galaxies: interactions

\section{Introduction}

Ram pressure of the inter-galactic medium in the dense environments of galaxy clusters and groups can significantly affect the interstellar content of galaxies. Together with tidal stripping and gas outflows due to galaxy mergers, ram pressure stripping is an efficient mechanism removing their gas component. This quenches the star formation. Local enhancement of the gas density by the ram pressure, however, can increase the star formation rate in the central disk regions and in the tail of stripped material (Kronberger et al. 2008). Current observations showing gaseous tails and extra-planar gas (e.g. Crowl et al. 2005; Chung et al. 2007), and a number of numerical simulations (e.g. Vollmer et al. 2001; Roediger \& Brüggen 2007) confirm the importance of gas stripping in the evolution of galaxies. Although some gas tails could be due to tidal interactions between galaxies in the cluster (Chung et al. 2007), these are rapidly destroyed by the ram pressure (Kapferer et al. 2008). Ram pressure then creates its own long gas tails (Roediger \& Brüggen 2008).

When the galaxy plunges into the intracluster medium (ICM), a complex hydrodynamical interaction of the interstellar matter (ISM) and ICM takes place. Several phases of ISM-ICM interaction were recognized by Roediger \& Brüggen (2006): the initial ram pushing of the ISM out of the disk, followed by stripping of elements accelerated to the escape velocity, in a background supported by continuous stripping. A simple estimate of the stripping amount suggests that if the ram pressure of the wind exceeds the local gravitational restoring force per unit area, the gas is released from the potential of the galaxy (Gunn \& Gott 1972, GG72). This approximation works well for face-on cases with constant long-lasting flows of the ICM. However, in real situations, galaxies experience a time-varying ram pressure due to their evolving orbital velocity and radially changing ICM density. An approximative criterion where the stripping is proportional to the momentum of the ICM encountered along the orbit was introduced by Jáchym et al. (2007).

A long-term ram pressure corresponding to the overall cluster ICM density profile can be accompanied by short pressure spikes from local clumps in the ICM distribution or wakes caused by previous stripping events. Tonnesen \& Bryan (2008) studied the role of ICM overdensities and their infalling motions, and suggested that even small substructures in the ICM may evoke a stronger stripping than that corresponding to the given position in the cluster. In short ICM-ISM encounters, not all the ISM is accelerated to the escape speed and its re-accretion can occur. Very recently, Tonnesen \& Bryan (2009) took into 
account the multi-phase nature of the ISM, and found different results, according to the clumpiness of the gas. Vollmer et al. (2009) argue that ram pressure is more efficient on ionized gas, and they see evidence of differential ram pressure efficiency according to the nature of the multiphase gas.

The question of how the orientation of the galaxy relative to the orbital direction influences the final stripping was touched on by many authors (Quilis et al. 2000; Vollmer et al. 2001; Schulz \& Struck 2001; Hidaka \& Sofue 2002; Marcolini et al. 2003; Roediger \& Brüggen 2006). For tilted galaxies the effect of the hydrodynamical shielding is more pronounced in comparison to the face-on case and yields weaker stripping. Moreover, due to galactic rotation, near edge-on ram pressures evoke asymmetric shapes of the ISM disks (e.g. Roediger \& Brüggen 2006). From previous simulations, only close-to edge-on orientations were identified as less efficient in the stripping than face-on cases (Quilis et al. 2000; Roediger \& Brüggen 2006). The tilted stripping was found to be delayed compared to face-on (Vollmer et al. 2001), and takes a longer time (Farouki \& Shapiro 1980). Other features, like tails of stripped material forming on the disk side where the gas rotates against the wind (Phookun \& Mundy 1995), or annealing of inclined galaxies by a stronger loss of angular momentum (Schulz \& Struck 2001), were noticed. Mimicking the hydrodynamical shielding of tilted galaxies with a simple geometrical prescription, Vollmer et al. (2001) performed a set of sticky-particle simulations with time-varying ram pressure peaks. Roediger \& Brüggen (2006, 2007), using hydrodynamical adaptive-mesh-refinement simulations, introduced models of galaxies experiencing a constant ICM flow in a periodic boundary box, or orbiting on realistic orbits in clusters.

What is the role of the galactic disk tilt angle in various distributions of the ICM, and could a simple dependence between the stripping amount and the tilt angle be found from simulations? To answer these questions we perform in this paper an extensive grid of Smoothed particle hydrodynamics (SPH) simulations with time-varying large or small ram pressure peaks of generally tilted ram pressure stripping events. This completes other existing simulations based on different numerical approaches (AMR, sticky-particles etc.) and follows our simulations on the face-on study made by Jáchym et al. (2007). Rather than describing in detail the process of stripping itself (that shall be studied in a forthcoming paper), we focus on the quantitative outcomes of the tilted ISM-ICM interactions. To isolate the effect of the disk inclination, we deal with galaxies on strictly radial orbits freely falling from the cluster outskirts. This condition however does not imply a strong limitation since ram pressure profiles experienced by galaxies along slightly elliptical orbits may be modeled with strictly-radial ones through an ICM distribution with a lower value of the central density and a lower concentration towards the center. With the results of the simulation grid in hand, we use a simplified test particle model to separate the various effects taking place in the ISM-ICM interactions, and to search for a simple dependence that would fit the numerical results.

Our model, initial conditions and simulation method are introduced in Sect. 2. The results of simulations are presented and analyzed in Sects. 3 and 4. Their comparison with a simple test model is discussed in Sect. 5. How can the results of the tilted stripping be estimated? This question is answered in Sect. 6, which deals with a momentum delivery criterion. In the discussion of Sect. 7, we compare our results with outcomes of previous numerical simulations.

\section{Modeling}

\subsection{Galaxy model}

Our model galaxy is a late-type massive galaxy (LM-type in Jáchym et al. 2007): a three-component system with Plummer bulge and dark halo, and with Toomre-Kuzmin- $\operatorname{sech}^{2}\left(z / z_{0}\right)$ disk. The values of the model parameters are $M_{\mathrm{d}}=8.6 \times 10^{10} M_{\odot}$, $a_{\mathrm{d}}=4 \mathrm{kpc}, z_{0}=0.25 \mathrm{kpc}, M_{\mathrm{b}}=1.3 \times 10^{10} M_{\odot}, a_{\mathrm{b}}=0.4 \mathrm{kpc}$, and $M_{\mathrm{h}}=4.2 \times 10^{11} M_{\odot}, a_{\mathrm{h}}=20 \mathrm{kpc}$. All three components are represented with particles (12000 for the stellar disk, 12000 for the halo, and 6000 for the bulge). Their distribution is truncated at $16 \mathrm{kpc}$ (disk), $4 \mathrm{kpc}$ (bulge), and $40 \mathrm{kpc}$ (halo). The corresponding rotation curve is flat with a velocity of about $250 \mathrm{~km} \mathrm{~s}^{-1}$. The ISM in the disk follows the same density profile as the stellar component with the same radial scale $\left(a_{\mathrm{d}}=4 \mathrm{kpc}\right)$. Its mass forms $10 \%$ of the total disk mass. The ISM disk consists of $12000 \mathrm{SPH}$ particles which provides sufficient resolution for the large-scale effects of interest in this paper.

\subsection{Cluster model}

The galaxy cluster is modeled with dark matter (DM) and ICM gas. Their volume densities follow a $\beta$-profile (Cavaliere $\&$ Fusco-Femiano 1976; Schindler et al. 1999): $\rho=\rho_{0}(1+$ $\left.R^{2} / R_{\mathrm{c}}^{2}\right)^{-3 \beta / 2}$. For the standard cluster model we use $\beta_{\mathrm{ICM}}=1 / 2$, $\rho_{0, \mathrm{ICM}}=6.5 \times 10^{-3} \mathrm{~cm}^{-3}=0.64 \times 10^{-26} \mathrm{~g} \mathrm{~cm}^{-3}$ and $R_{\mathrm{c}, \mathrm{ICM}}=$ $13.4 \mathrm{kpc}$. The fixed DM, which provides the gravitational potential, has $\rho_{0, \mathrm{DM}}=3.8 \times 10^{-4} M_{\odot} \mathrm{pc}^{-3}, R_{\mathrm{c}, \mathrm{DM}}=320 \mathrm{kpc}$, and $\beta_{\mathrm{DM}}=1$. The ICM is introduced with $120000 \mathrm{SPH}$ particles distributed within a $140 \mathrm{kpc}$ truncation radius about the cluster center. Initially, the ICM particles are assigned velocity dispersions calculated from the hydrostatic equilibrium equation of the cluster. The system then slightly relaxes to a stable state. Particles that reach the truncation radius of $140 \mathrm{kpc}$ are relocated to the centrally symmetric position. See Jáchym et al. (2007) for more details.

\subsection{Initial conditions}

The model galaxy initially starts with zero velocity from the cluster periphery $(R=1 \mathrm{Mpc})$ and freely falls along a strictly radial orbit towards the cluster center. In the standard model the galaxy reaches the center at time $T=1.64 \mathrm{Gyr}$ with a velocity of about $1300 \mathrm{~km} \mathrm{~s}^{-1}$. The simulation is followed until $T=2 \mathrm{Gyr}$ when the galaxy has already left the central part of the cluster filled with ICM particles. Thus, each simulation run corresponds to one orbit of the galaxy through the cluster center.

To treat various cluster environments, we vary the values of the $R_{\mathrm{c}, \mathrm{ICM}}$ and $\rho_{0, \mathrm{ICM}}$ parameters, multiplying each standard value by factors of $8,4,1$, and 0.25 . Table 1 defines the basic set of simulation runs which are labeled with the corresponding factors ( 0 stands for the factor of 0.25 ). Table 1 gives for each run the combination of $R_{\mathrm{c}, \mathrm{ICM}}$ and $\rho_{0, \mathrm{ICM}}$ parameters, the peak value of the ram pressure along the orbit, and the column density of the ICM encountered on the orbit. Note that narrow ICM distributions or those with low values of density may represent ICM overdensities or debris structures left over in the cluster from recent stripping events.

Abadi et al. (1999) warned against too massive ICM particles that can punch holes in the gas disk and cause a large artificial drag on the ISM particles. In our standard model $(R 1 \rho 1)$ one ICM particle has a mass of about $5 \times 10^{5} M_{\odot}$, which is almost equal to the mass of an individual ISM particle. Concerning the 
Table 1. Set of basic simulation runs. We refer to $R 1 \rho 1$ as to the standard run. Values of $\Sigma_{\mathrm{ICM}}$ correspond to $R_{\mathrm{ICM}}^{\text {trunc }}=140 \mathrm{kpc}$.

\begin{tabular}{|c|c|c|c|}
\hline run & $\begin{array}{c}R_{\mathrm{c}, \mathrm{ICM}}, \rho_{0, \mathrm{ICM}} \\
\left(\mathrm{kpc}, 10^{-3} \mathrm{~cm}^{-3}\right)\end{array}$ & $\begin{array}{c}p_{\mathrm{ram}}^{\max } \\
\mathrm{cm}^{-3}\left(\mathrm{~km} \mathrm{~s}^{-1}\right)^{2} \\
\end{array}$ & $\begin{array}{c}\Sigma_{\mathrm{ICM}} \\
\left(M_{\odot} \mathrm{pc}^{-2}\right)\end{array}$ \\
\hline$R 4 \rho 8$ & $53.6,52$ & 100913 & 114 \\
\hline$R 4 \rho 4$ & $53.6,26$ & 46752 & 57 \\
\hline$R 4 \rho 1$ & $53.6,6.5$ & 10993 & 14 \\
\hline$R 4 \rho 0$ & $53.6,1.6$ & 2705 & 3.6 \\
\hline$R 1 \rho 8$ & $13.4,52$ & 89214 & 41 \\
\hline$R 1 \rho 4$ & $13.4,26$ & 43827 & 20 \\
\hline$R 1 \rho 1$ & $13.4,6.5$ & 10810 & 5 \\
\hline$R 1 \rho 0$ & $13.4,1.6$ & 2693 & 1.3 \\
\hline$R 0 \rho 8$ & $3.4,52$ & 86549 & 12 \\
\hline$R 0 \rho 4$ & $3.4,26$ & 43143 & 5.9 \\
\hline$R 0 \rho 1$ & $3.4,6.5$ & 10769 & 1.5 \\
\hline$R 0 \rho 0$ & $3.4,1.6$ & 2689 & 0.4 \\
\hline
\end{tabular}

ICM component setup, its truncation radius of $140 \mathrm{kpc}$ was chosen so that ram pressure in the standard cluster exceeds at that distance the gravitational restoring force in the disk outskirts of an approaching galaxy.

We introduce the tilt angle of the disk with respect to the orbital direction of the galaxy as $i=0^{\circ}$ for edge-on and $i=90^{\circ}$ for face-on orientation, throughout the paper.

\subsection{Simulation method}

The simulations were carried out with the tree/SPH code GADGET (Springel et al. 2001) adapted by Jáchym et al. (2007) for calculations of ISM-ICM interactions. Smoothed particle hydrodynamics (SPH, Lucy 1977; Gingold \& Monaghan 1977) in its standard formulation has significant problems with contact discontinuities where the density jump is very large. Across such contact discontinuities, fluid instabilities can be suppressed (e.g. Agertz et al. 2007), or other artifacts can occur when the sampling is poor (e.g. Okamoto et al. 2003). The problem of ICMISM interaction is basically a special case of this. Jáchym et al. (2007) modified GADGET to estimate smoothing lengths of either ICM or ISM particles separately from neighbors of a corresponding phase. This means that the search of the neighboring particles continues until a desired number of proper neighbors are found, while ignoring those of the second phase. It ensures that even a moderate number of ICM particles will fully cover during the interaction the ISM disk, as the ICM particles do not shrink when approaching the dense ISM in the disk. This modified code was used by Jáchym et al. (2007) for simulations of face-on ram pressure stripping events.

Although large ICM particles result in a low spatial resolution and ISM particles that lack pressure gradients from the wind, our suggestion provides a simple way to avoid the above limitation of the standard SPH method, and it may be used for the quantitative purposes of this paper. An ideal solution could involve a new self-consistent way to estimate densities that allows a better representation of contact discontinuities - e.g. based on a tessellation scheme (recently published by Springel 2009), implementation of the particle splitting mechanism (Kitsionas \& Whitworth 2007) into the standard SPH, or the new SPH scheme of Kawata et al. (2009).

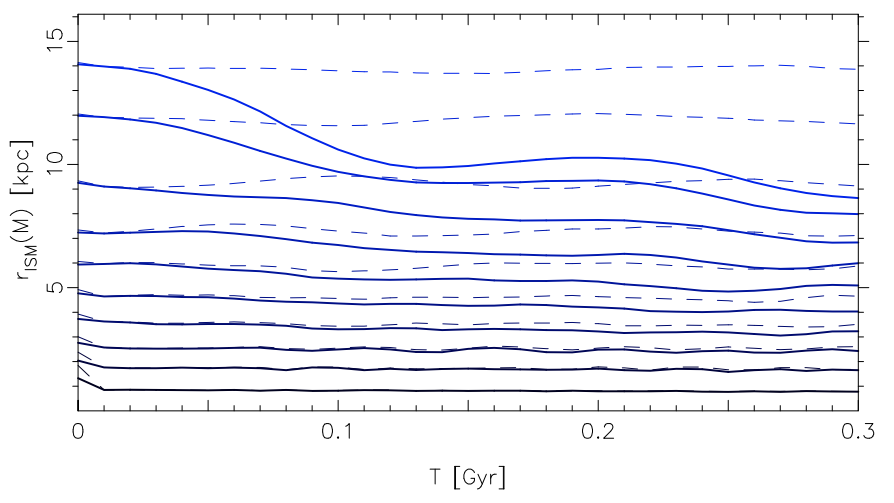

Fig. 1. Zero-order test vs. isolated evolution: Disk radii encompassing $95 \%, 90 \%, 80 \%, \ldots, 10 \%$ of the total ISM mass (from top down) as functions of time in the case of the galaxy evolving at rest in the center of the standard cluster (solid curves), and in an isolated galaxy (dashed).

\subsection{Zero-order simulation run}

In order to test the influence of the simulation technique used, and the effect of resolution on the results of our stripping simulations, we present a zero-order test: The galaxy is placed at rest at the center of the cluster and left to evolve surrounded by the ICM for about $300 \mathrm{Myr}$ (the time comparable to the duration of the standard ISM-ICM interaction discussed later). The evolution of the galaxy's gaseous disk is displayed in Fig. 1 showing radii that encompass fixed fractions of the total ISM mass (solid curves) evolving with time. The ISM disk (or at least its outer layers) is compressed by an abrupt introduction of the static external pressure of the ICM on a time scale of about 100 Myr. After this period the outer layers start to slightly oscillate while the disk slowly continues to settle towards an equilibrium. All the ISM particles during the simulation stay bound to the galaxy. The stellar disk component is unchanged during the simulation. For comparison, the evolution of the ISM disk of an isolated galaxy, not exposed to any external pressure, is plotted in Fig. 1 with dashed curves.

The ISM component is treated isothermally in our simulations. This simple approach is used to roughly mimic an efficient radiative cooling of the ISM gas. The disk confinement in Fig. 1 may thus be understood in terms of an abrupt increase of the external pressure after the galaxy has been placed into the ICM due to its isothermality the ISM cannot heat up (to compensate the increased external pressure) and is compressed instead. In Fig. 1, an extreme effect is shown due to a maximum pressure corresponding to the cluster center. However, in the ram pressure stripping simulations that we deal with further in this paper, the galaxy arrives at the cluster center only gradually from its outskirts. This aspect will be treated in the following section.

Concerning the influence of our numerical technique on the simulation outputs, no Rayleigh-Taylor instabilities or spurious effects due to the lack of resolution appeared in the zero-order test simulation.

\section{Process of stripping}

For non-face-on orientations of the galaxy with respect to the ICM wind, the galactic rotation starts to play a role. Its effect is most pronounced for near edge-on cases when it evokes an asymmetry in the gaseous disk. This can be seen in Fig. 2 where snapshots of the galaxy passing edge-on through the center of a wide $R 4 \rho 1$ cluster are depicted. (For illustrative purposes the $R 4 \rho 1$ run is studied here rather than the standard one where 

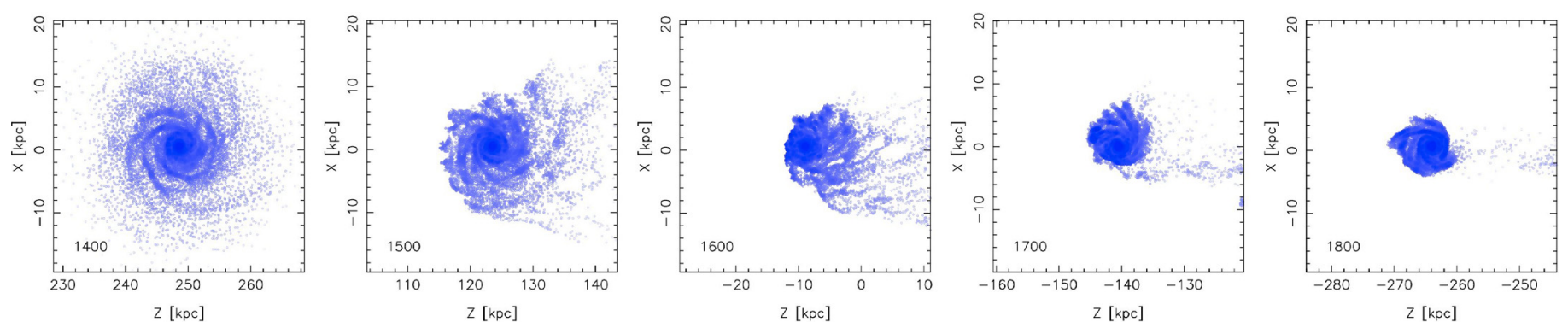

Fig. 2. Pole-on views of the ISM disk crossing edge-on through the $R 4 \rho 1$ cluster. The disk rotates clockwise; the ram pressure operates from the left. Color saturation corresponds to increasing ISM density.

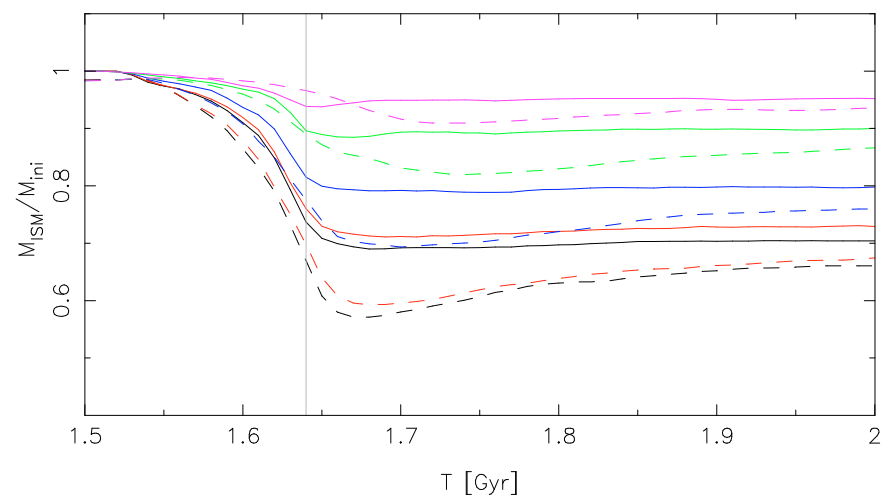

Fig. 3. Effect of the inclination: Evolution of the ISM mass bound to the galaxy (solid curves) or enclosed within an evaluation cylinder around the disk plane (dashed) in simulations with disk tilt decreasing from face-on to edge-on (from bottom up) of the standard $R 1 \rho 1$ run.

edge-on stripping is less prominent.) Due to rotation, the disk side moving parallel to the wind (upper side in Fig. 2) experiences ram pressure that is lower by a factor of $\left(v-v_{\text {rot }}\right)^{2} /\left(v+v_{\text {rot }}\right)^{2}$ with respect to the opposite disk side; $v$ is the orbital speed of the galaxy and $v_{\text {rot }}$ is the rotational velocity of the disk. However, as e.g. the second panel of the figure shows, the parallel-rotating side is more severely stripped than the opposite one and it is thus not the highest ram pressure that yields the strongest stripping. This paradox was noted by Roediger \& Brüggen (2006) as well. The explanation is in terms of time delay: on the parallel-rotating side, the gas is accelerated towards more rotational velocity and exits the galaxy almost at once. At the opposite side, the gas is being braked from $v-v_{\text {rot }}$ towards $v$ and with lower or no centrifugal force it has time to fall towards the center of galaxy. Then, it still has to rotate half a turn around the galaxy, and it has time to be re-accreted (or at least it stays around the galaxy for a longer time). Snapshots in Fig. 2 further show how, due to rotation, the tail of the stripped material winds up around the disk.

To measure the actual level of stripping we set up a cylindrical zone about the galactic disk plane $(r=16 \mathrm{kpc}$ and $|z| \leq 3$ $\mathrm{kpc}$ ), and follow the enclosed ISM mass and the flow of the material through the zone boundary. This is shown in Figs. 3 and 4 for five simulations of the standard run with different tilt angles, together with the ISM mass fraction bound to the galaxy (solid curves in Fig. 3). It confirms the expected trend: with the tilt angle growing towards face-on, more material is stripped. The maximum departure of the curves of the bound and in-the-zoneenclosed ISM mass in Fig. 3 indicates the amount of material that is re-accreted after the ISM-ICM interaction. The bound mass in Fig. 3 is given by the sum of the ISM particles for which $E_{\mathrm{kin}}+E_{\mathrm{pot}}<0$. Note that the system of the galaxy is not closed - it experiences a changing effect of the external potential of

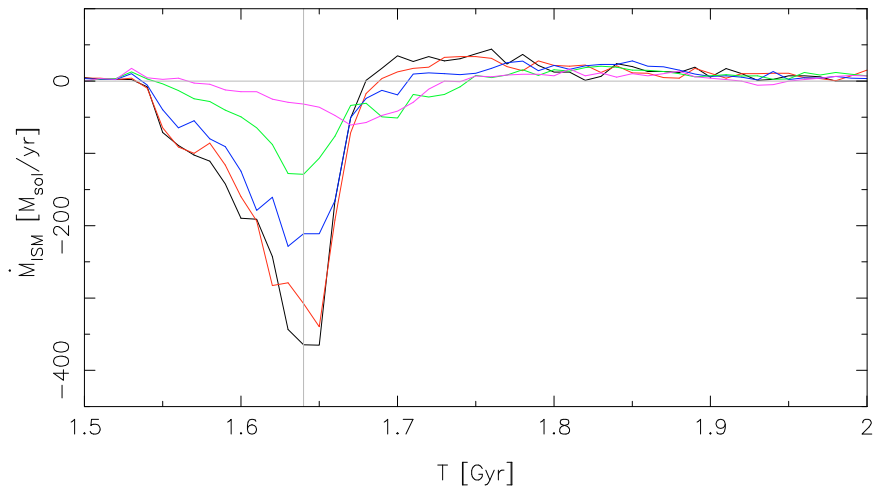

Fig. 4. Mass flow of the ISM through the boundary of the evaluation cylinder for five simulations of Fig. 3 .

the cluster and an external pressure. A slight growth of the solid curves in Fig. 3 may be found at later simulation times.

The "stripping rate" $\dot{M}_{\mathrm{ISM}}$, i.e. the flow of the ISM through the boundary of the evaluation zone, exceeds almost $400 M_{\odot} \mathrm{yr}^{-1}$ for a face-on galaxy, the peak value decreases towards the edgeon orientation to about $50 M_{\odot} \mathrm{yr}^{-1}$ (see Fig. 4). This figure shows not only the removal rate $\left(\dot{M}_{\mathrm{ISM}}<0\right)$ but also a reversed reaccretion rate of the shifted material $\left(\dot{M}_{\text {ISM }}>0\right)$ - in the face-on case it reaches about $50 M_{\odot} \mathrm{yr}^{-1}$ after the passage of the galaxy through the central cluster part. (Note that the amount of reaccretion is dependent on the extent of the evaluation zone.)

The re-accretion process may be better followed in Fig. 5 which traces the actual distances of the bound ISM behind the galaxy (in the direction of the wind) for face-on and edge-on cases. In the face-on orientation, the ISM is by an increasing ram pressure first gradually pushed out of the disk plane, and then rapidly "thrown" to large distances, shortly after the cluster center passage. This material returns to the disk at later simulation times. In the edge-on orientation, the disk is first compressed by the rising ram pressure, and then some material is shifted to larger distances. The peak in Fig. 5 is, however, much smaller than in the face-on case. The reason why in near edge-on orientations the pushed material stays close to the disk is in the conservation of the angular momentum of the ISM particles rotating in the disk. When pushed by the ram pressure, the particles wind up around the disk. We checked this behavior in a more efficient run $R 4 \rho 1$ where almost the same amount of ISM is stripped as in the standard face-on run.

Apart from the total stripped amount, no radical differences in the duration of the stripping process of variously tilted galaxies can be seen from our simulations. Several details, like a slight time shift of the maximum of the stripping rate, or the course of re-accretion, look different in edge-on and face-on orientations, however these effects seem to be of secondary nature. 
Table 2. List of performed simulations - results. From left to right: fraction of the stripped ISM mass for simulations with decreasing inclination, stripped mass fraction estimate of GG72 criterion, corresponding stripping radii and the estimate of GG72.

\begin{tabular}{lcccccccccccc}
\hline \hline run & $\begin{array}{c}M_{\text {strip }}^{90^{\circ}} \\
(\%)\end{array}$ & $\begin{array}{c}M_{\text {strip }}^{70^{\circ}} \\
(\%)\end{array}$ & $\begin{array}{c}M_{\text {strip }}^{45^{\circ}} \\
(\%)\end{array}$ & $\begin{array}{c}M_{\text {strip }}^{20^{\circ}} \\
(\%)\end{array}$ & $\begin{array}{c}M_{\text {strip }}^{0^{\circ}} \\
(\%)\end{array}$ & $\begin{array}{c}M_{\text {strip }}^{\mathrm{GG72}} \\
(\%)\end{array}$ & $\begin{array}{c}r_{\text {strip }}^{90^{\circ}} \\
(\mathrm{kpc})\end{array}$ & $\begin{array}{c}r_{\text {strip }}^{70^{\circ}} \\
(\mathrm{kpc})\end{array}$ & $\begin{array}{c}r_{\text {strip }}^{45^{\circ}} \\
(\mathrm{kpc})\end{array}$ & $\begin{array}{c}r_{\text {strip }}^{20^{\circ}} \\
(\mathrm{kpc})\end{array}$ & $\begin{array}{c}r_{\text {strip }}^{0^{\circ}} \\
(\mathrm{kpc})\end{array}$ & $\begin{array}{c}r_{\text {strip }}^{\mathrm{GG72}} \\
(\mathrm{kpc})\end{array}$ \\
\hline$R 4 \rho 8$ & 93 & 91 & 89 & 86 & 70 & 93 & 1.5 & 0.9 & 1.4 & 0.8 & 1.2 & 1.5 \\
$R 4 \rho 4$ & 85 & 85 & 80 & 72 & 52 & 83 & 1.6 & 1.8 & 1.6 & 1.6 & 2.0 & 2.4 \\
$R 4 \rho 1$ & 59 & 56 & 48 & 34 & 24 & 57 & 2.5 & 2.6 & 2.6 & 3.7 & 5.1 & 4.5 \\
$R 4 \rho 0$ & 26 & 25 & 18 & 10 & 6 & 36 & 6.0 & 6.2 & 6.5 & 6.5 & 7.1 & 6.8 \\
$R 1 \rho 8$ & 79 & 77 & 70 & 54 & 36 & 92 & 1.5 & 1.6 & 1.6 & 2.2 & 2.7 & 1.6 \\
$R 1 \rho 4$ & 63 & 61 & 50 & 30 & 19 & 81 & 2.1 & 2.5 & 3.1 & 3.2 & 4.0 & 2.5 \\
$R 1 \rho 1$ & 30 & 27 & 20 & 10 & 5 & 57 & 5.5 & 6.3 & 6.5 & 7.2 & 8.0 & 4.5 \\
$R 1 \rho 0$ & 9 & 8 & 6 & 2 & 1 & 36 & 8.6 & 9.0 & 9.3 & 9.9 & 10.3 & 6.8 \\
$R 0 \rho 8$ & 37 & 35 & 27 & 13 & 7 & 92 & 5.5 & 4.6 & 6.1 & 6.5 & 7.2 & 1.6 \\
$R 0 \rho 4$ & 22 & 21 & 14 & 6 & 3 & 81 & 6.8 & 7.7 & 8.0 & 9.2 & 9.2 & 2.5 \\
$R 0 \rho 1$ & 5 & 5 & 3 & 1 & 1 & 57 & 9.8 & 9.9 & 10.2 & 10.6 & 10.7 & 4.5 \\
$R 0 \rho 0$ & 1 & 1 & 0 & 0 & 0 & 36 & 11.1 & 11.1 & 11.2 & 11.4 & 11.5 & 6.8 \\
\hline
\end{tabular}

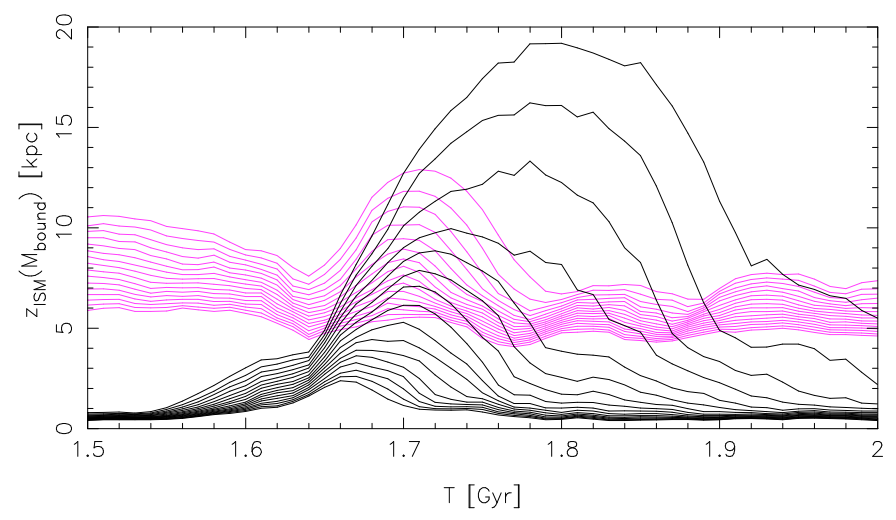

Fig. 5. ISM re-accretion: Distances behind the galaxy (in the direction of the wind) within which a certain fraction of actually bound ISM particles are enclosed (from $95 \%$ to $80 \%$, curves from top down). Face-on (black) and edge-on simulations of $R 1 \rho 1$ run. (In the edge-on case the distances are taken in absolute value as initially one half of the particles occur ahead of the galactic mid-plane.)

\subsection{Static vs. dynamic ICM pressure}

How does the effect of the disk radius reduction due to ram pressure compete with the disk confinement due to the static ICM pressure discussed in Sect. 2.5? In Fig. 6 the rate of the disk radius reduction caused by the ram pressure stripping in the standard face-on run is compared with the upper curve of Fig. 1. It shows that the effect of the ram pressure is more important, even though the zero-order test took place in the center of the standard cluster where the disk experienced the maximum external pressure possible. In the ram pressure simulation the in-flying galaxy enters the ICM distribution at the truncation radius where the ICM density (and its pressure) is only small, and plunges deeper towards the cluster center, which is crossed very fast. Thus the actual role of the static ICM pressure will be even less than in Fig. 6.

Although the disk confinement due to the static ICM pressure operates against ram pressure - it does not strip the gas but makes the disk more resistant to the ram pressure - the tendency of the increasing effect of the static ICM pressure in "larger" clusters goes in the same direction as the effect of ram pressure stripping. Recently, Tonnesen \& Bryan (2009) have taken into account the multi-phase nature of the gas disk and found that the stripping radius is considerably smaller for galaxies with

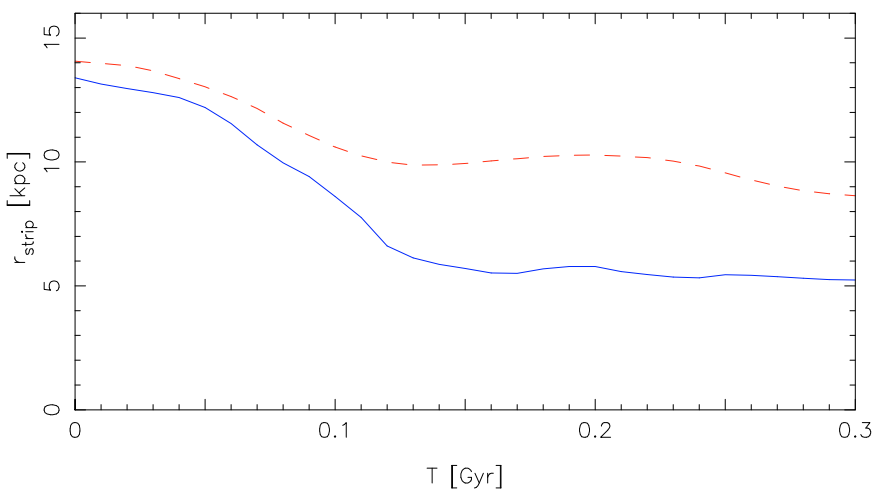

Fig. 6. Ram pressure stripping vs. zero-order test: Actual estimate of the stripping radius of the face-on galaxy in the standard $R 1 \rho 1$ cluster (solid) as a function of time, vs. radius of the disk confined by static ICM pressure (dashed, the $95 \%$ radius from Fig. 1).

radiative cooling, although the amount of stripping is similar to that in galaxies without cooling. This goes in the same direction as the above effect observed in our simulations.

We conclude that the disk confinement due to static external ICM pressure is much less important than the stripping due to ram pressure and we neglect this effect in analyzing the simulations in the following sections.

\section{Results}

In this section the results of the basic set of simulation runs introduced in Table 1 are described. For each run listed we have performed five simulations with increasing disk inclination: $i=0^{\circ}$ (edge-on), $20^{\circ}, 45^{\circ}, 70^{\circ}$ and $90^{\circ}$ (face-on). The overall Table 2 provides the results: mass fraction of the stripped material $\left(M_{\text {strip }}\right)$ at the end of the simulation for five disk inclinations, stripped mass fraction estimated with the GG72 formula, stripping radii $r_{\text {strip }}$ for five inclinations, and the stripping radius corresponding to GG72.

\subsection{Stripped amount}

The fraction of the ISM particles that is no longer bound to the galaxy at the final simulation time yields the stripped mass fractions, whose dependence on inclination is shown in Fig. 7. The 


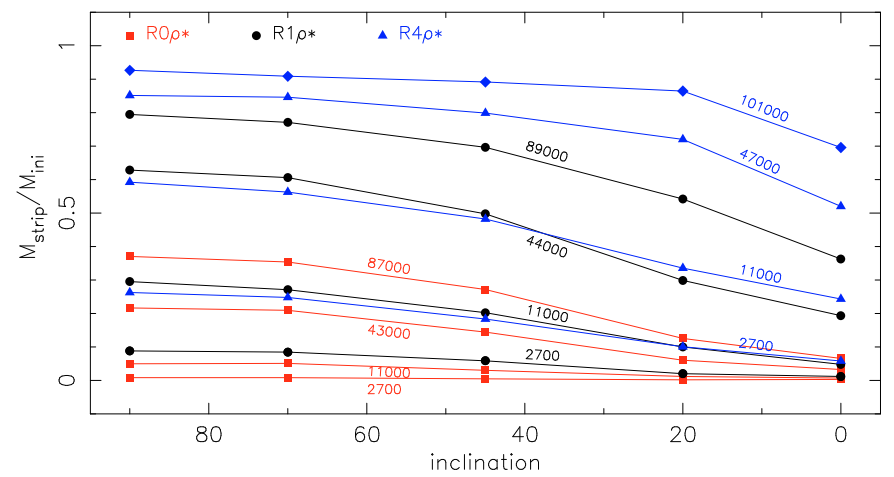

Fig. 7. Stripped mass fraction as a function of disk inclination for runs from Table 2. Each run is labeled with the peak value of the ram pressure. The inclination is $0^{\circ}$ for edge-on and $90^{\circ}$ for face-on orientation.

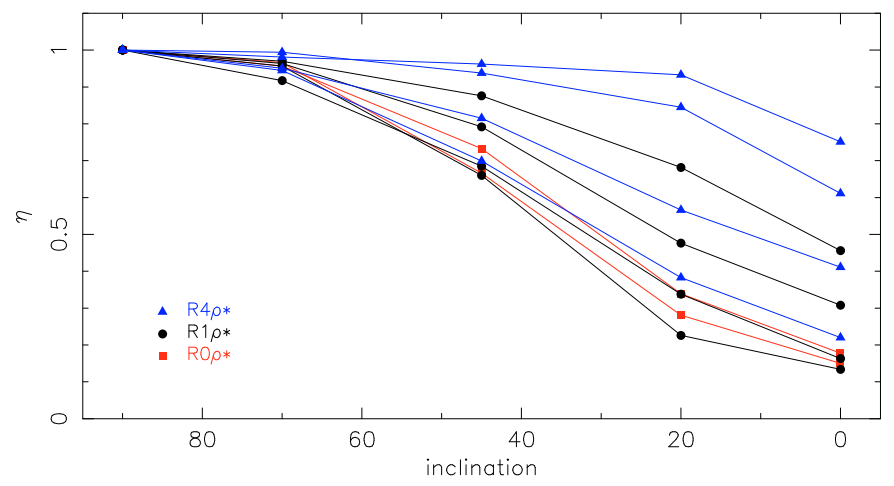

Fig. 8. Stripping efficiency with respect to inclination $\eta=$ $M_{\text {strip,i }} / M_{\text {strip, } 90^{\circ}}$ as a function of the inclination (runs $R 0 \rho 1$ and $R 0 \rho 0$ are not plotted).

general tendency of the plot is evident - with the inclination decreasing from face-on to edge-on, the amount of stripping declines. Furthermore:

- Previous simulations of Quilis et al. (2000), Roediger \& Brüggen (2006) and others have shown that there is almost no difference between $i=70^{\circ}$ and face-on stripping $\left(i=90^{\circ}\right)$. This is true in Fig. 7 for all runs.

- For large pressure peaks (runs $R 4 \rho 4$ and $R 4 \rho 8$ ), the stripping amount is almost independent of the inclination (for angles higher than about $20^{\circ}$ ).

- The dependence of the stripping amount on inclination is more pronounced for smaller ram pressure peaks.

- Runs with the same value of $R_{\mathrm{c}, \mathrm{ICM}} \cdot \rho_{0, \mathrm{ICM}}$ quantity show close profiles of the $M_{\text {strip }}(i)$ curves - e.g. $(R 4 \rho 1$ and $R 1 \rho 4)$, or $(R 4 \rho 0, R 1 \rho 1$, and $R 0 \rho 4)$.

The behavior with varying inclination is more clearly displayed in Fig. 8 which shows a new parameter $\eta$ that we name the stripping efficiency with respect to inclination. It is the ratio of the stripped mass fraction relative to the face-on case:

$\eta(i)=\frac{M_{\mathrm{strip}, i}}{M_{\mathrm{strip}, 90^{\circ}}}=\frac{1-M_{\mathrm{fin}, i} / M_{\mathrm{ini}}}{1-M_{\mathrm{fin}, 90^{\circ}} / M_{\mathrm{ini}}}$.

Thus, $\eta$ characterizes the relative strength of a given ram pressure profile to strip the ISM from an inclined galaxy with respect to the face-on case. In Fig. 8, one observes that the stripping efficiency always declines with decreasing inclination angle. Moreover, both wider and higher ram pressure peaks yield higher efficiencies. For smaller pressure peaks, $\eta$ falls fastest

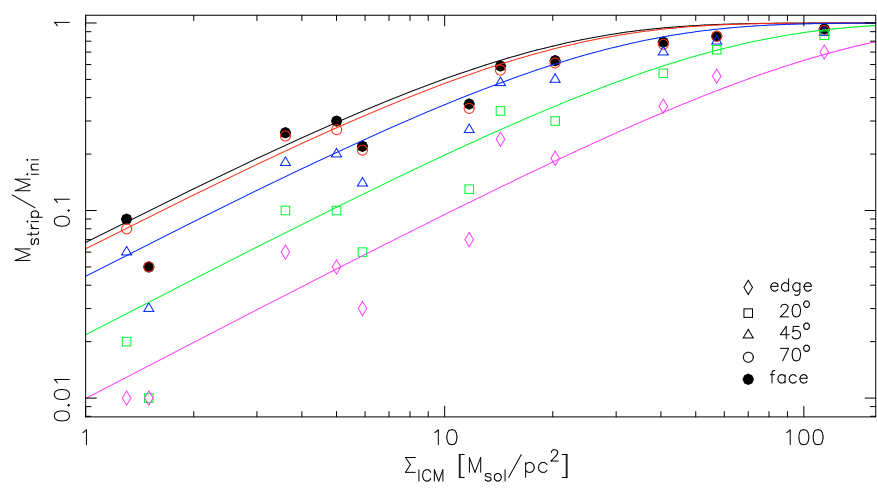

Fig. 9. Stripped mass fraction as a function of the column density of the encountered ICM. The symbols denote the results of the SPH simulations for various inclinations. The curves correspond to the fitting curve in Eq. (2).

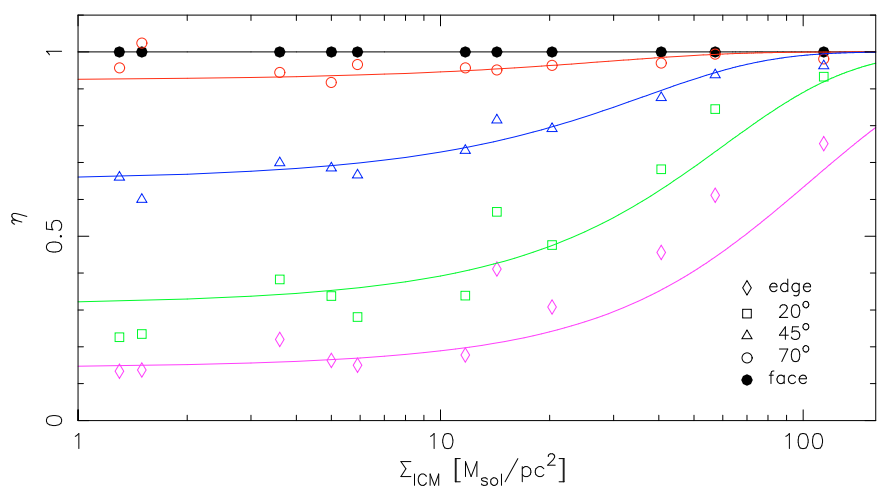

Fig. 10. Stripping efficiency with respect to inclination as a function of the column density of the encountered ICM. The curves correspond to the fitting curve in Eq. (2).

at medium inclinations (between $20^{\circ}$ and $70^{\circ}$ ), while for large peaks at inclinations smaller than $20^{\circ}$.

Stripping of face-on galaxies depends on the amount of the ICM encountered along the trajectory (Fig. 15 in Jáchym et al. 2007): galaxies crossing clusters with such combinations of $R_{\mathrm{c}, \mathrm{ICM}}$ and $\rho_{0, \mathrm{ICM}}$ parameters that $\Sigma_{\mathrm{ICM}} \sim$ const. were stripped to the same level. For inclined cases this hypothesis is tested in Figs. 9 and 10, which show the stripped mass fraction and the stripping efficiency with respect to inclination as a function of the column density of the encountered ICM, respectively. Different colors refer to simulations with different inclination angles. The following trends are apparent

- with increasing amount of encountered ICM the stripped mass fraction and the efficiency increase;

- there is a tendency for the results from each inclination to follow a certain close relationship, despite some systematic deviations due to the different ICM density distributions;

- for high $\Sigma_{\mathrm{ICM}}$, these relations saturate towards complete stripping;

- for lower ICM column densities, edge-on stripping is reduced with respect to face-on by a constant factor.

All these results indicate that the column density of the ICM encountered during the ISM-ICM interaction is the key parameter determining the stripping outcome. It is much more important than the maximum value of the ram pressure experienced along the orbit (GG72 criterion). 


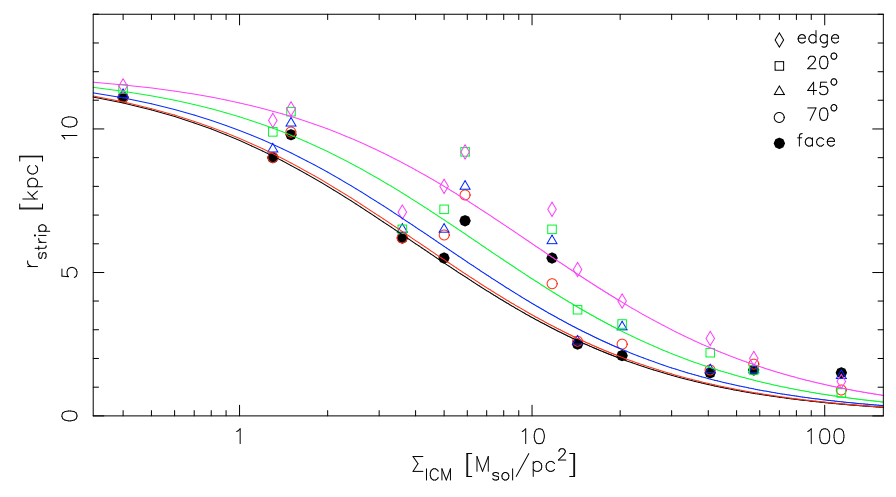

Fig. 11. Stripping radii $r_{\text {strip }}$ versus column density of the encountered ICM. The symbols are the same as in Fig. 9. The points are fitted with the fitting function in Eq. (3).

The numerical results in Fig. 9 can be fitted with the following formula

$\frac{M_{\text {strip }}}{M_{\text {ini }}}=1-\exp \left[-0.01 \Sigma_{\text {ICM }}\left(1+6 \sin ^{1.5} i\right)\right]$.

The exponent 1.5 provides a rather satisfactory match to the results with intermediate inclinations, as can be seen in both Figs. 9 and 10.

In Table 2, the stripped mass fraction estimated with the GG72 criterion is added. For the largest ram pressure peak $(R 4 \rho 8)$, the values match well the face-on results of the SPH simulations. For smaller peaks, however, GG72 overestimates the real stripped fraction. Of course, no dependence on the tilt angle is included in the GG72 criterion, and its multiplication by a simple cos $i$ term does not resolve the problem, as it yields zero stripping in edge-on cases.

\subsection{Stripping radii}

Due to an asymmetric shape of the gas disk that develops, especially after an inclined stripping event, the determination of its final radius, i.e. the stripping radius, is not trivial (see Roediger \& Brüggen 2006). In close to edge-on cases, all the stripped and shifted material stays in the plane of the disk and makes the determination difficult.

In Table 2, the given values of stripping radii correspond to radii within which $90 \%$ of the bound ISM is enclosed in the final simulation time. This may slightly underestimate the radius for the face-on orientation, while overestimating it for the edgeon case. The values do not show a strong dependence for large and small peak ram pressures; however, for medium pressure the trend of increasing stripping radii towards edge-on orientations is visible.

As shown in Fig. 11, the stripping radii for all simulations can be quite reasonably fitted with

$r_{\text {strip }}=12 \mathrm{kpc} /\left[1+\Sigma_{\mathrm{ICM}}(0.1+0.15 \sin i)\right]$.

The stripping radii estimated in the last column of Table 2 using the GG72 criterion show quite a good agreement for the largest run. However, there is a tendency to overestimate the SPH results. For smaller peaks, GG72 predicts less stripping corresponding only to the peak ram pressure.

\section{A simple test particle model}

In order to study the influence of the parameters more efficiently, and to separate various effects - the direct kinematics on the

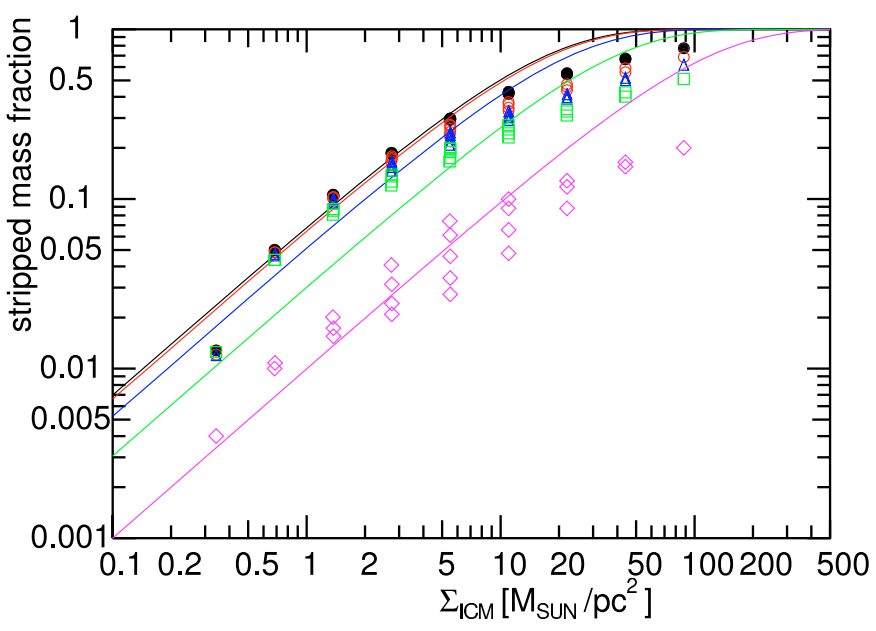

Fig. 12. The dependence of the stripped mass fraction on the column density of the swept-up ICM, for different tilt angles. The dots are results from a grid of simplified point-mass models, covering a range from $1 / 16$ to 16 times in both duration and strength of the ram pressure force pulse, centered about the value for our standard model. The curves are the analytical fit to the SPH results. The symbols and colors are identical to those of Fig. 9. Note that the range of parameters in both axes is much wider.

ISM induced by the ram-pressure, and the related hydrodynamical consequences - we construct a very simplified model for the removal of gas from a galaxy. The gas disk is represented by 10000 point masses distributed as in an exponential disk and held in the potential well composed of the bulge, stellar disk, and dark halo, with parameters identical to those used in the SPH computations. Initially, all these particles are in circular orbits about the center of the galaxy. Then, they are subjected to a Gaussian-shaped force pulse of specified tilt angle with respect to the galactic plane, duration, and maximum amplitude. The simulation starts at a time that is three times the pulse duration before the maximum. In the computation of the acceleration, the inertial mass of each particle is taken to be proportional to the gas surface density of the exponential disk, in order to account for the correct computation of the ram pressure in that part of the disk. For each particle, the equation of motion is integrated, and when the total energy becomes positive and the particle becomes unbound, it is marked and counted as having been stripped. If all particles were to experience this force pulse, the fraction of stripped particles is independent of the tilt angle, because all reach escape velocity within time-spans that depend little on their initial position in the galaxy and the force tilt angle.

In order to simulate the thickness of the disk, we introduce a shielding or shadowing of the particles downstream of those particles facing the force. In this, we follow the modeling by sticky particles by Vollmer et al. (2001). Particles that are shielded by at least one upstream particle are assumed to experience only the galaxy's internal forces. Experiments show that with the choice of a suitable value for the shadowing radius (typically $200 \mathrm{pc}$ ), we are able to reproduce well the amount of stripping for the standard model of the SPH computations, as well as its dependence on the tilt angle.

As is seen in Fig. 12, the stripped mass fractions from these simplified models behave in a very similar way as the SPH results. While at high values of the ICM column density the stripping is less severe and the relation is slightly flatter, the influence on the tilt angle is nearly identical - one obtains the same amount 


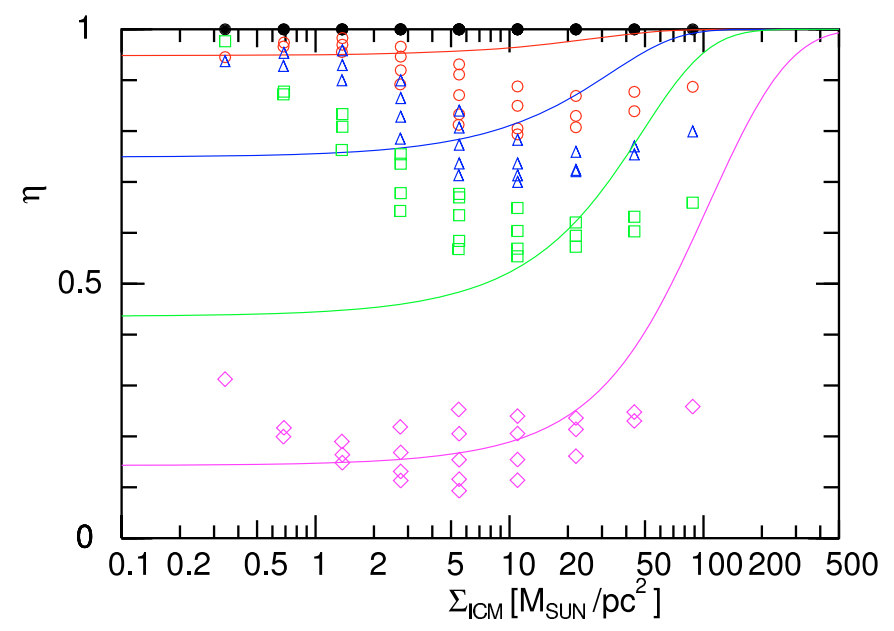

Fig. 13. Similar to Fig. 12, but for the dependence of the stripping efficiencies with respect to inclination as a function of the column density of the encountered ICM.

of stripping in the edge-on configuration as in the face-on case for an ICM column density that is larger by about a factor of 6 . This indicates that this modeling approach, and hence also the sticky-particle computations by Vollmer et al. (2001), appears to be quite reliable in capturing the main features of ram-pressure stripping. However, this simplified approach has notable differences to the full hydrodynamical approach, in features that we consider below.

The stripping efficiencies with respect to inclination (Fig. 13) show that the ratios of face-on and edge-on results are as large as from the SPH models, but that there are some limitations. First of all, at intermediate angles the simplified modeling gives results that are closer to the face-on case than we find in SPH. Furthermore, at high ICM column densities, one does not find such a steep increase of stripping as experienced in SPH. We searched quite extensively in parameter space, but could not find situations where the behavior matched the SPH results more closely. Evidently, this is a consequence of the limitations of the simplified treatment. We take this as an indication that this kind of diagram is sensitive to the different treatment of the (hydro)dynamics of the problem.

The simplified modeling does allow us to separate the different physical phenomena in the ram-pressure process. In particular it helps to identify the hydrodynamical shielding by the matter lying upstream as the principal cause for the dependence of stripping on tilt angle, seen in our SPH results. The deviations seen with respect to the SPH results suggest that the details of the behavior changes with tilt angle comes from the finer details of the hydrodynamics involved in the problem.

\section{Comparison with the analytical criterion}

From the consideration of the momentum provided by the instreaming ICM to the gas in the galactic disk Jáchym et al. (2007) derived the condition under which a volume element of disk gas (with column density $\Sigma_{\text {ISM }}$ for the face-on situation) would escape from a galaxy,

$\langle v\rangle_{\rho_{\mathrm{ICM}}} \Sigma_{\mathrm{ICM}} / \Sigma_{\mathrm{ISM}}=v_{\mathrm{after}}>v_{\mathrm{esc}}=\sqrt{-2 \Phi(r)}$,

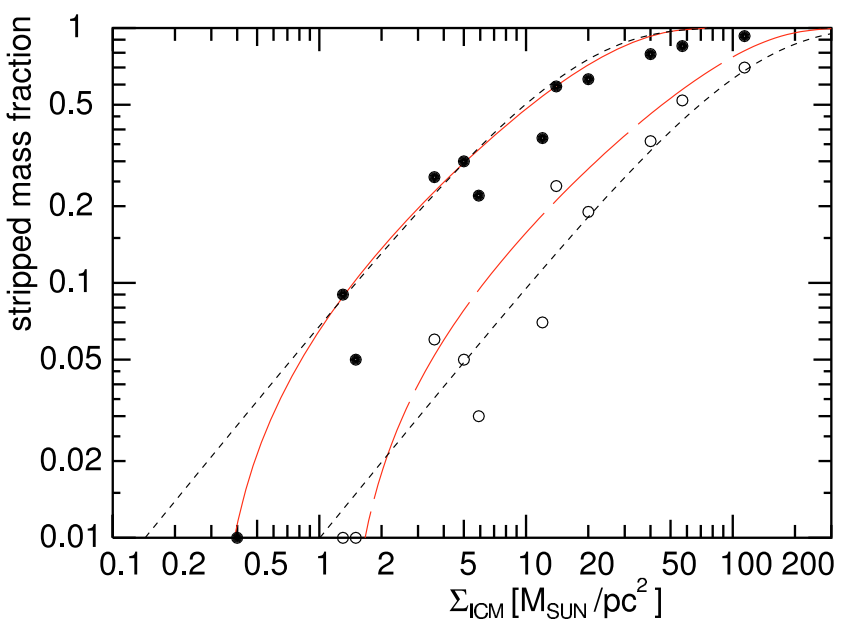

Fig. 14. The relation between stripped mass fraction and the column density of the swept-up ICM gas, computed for our standard model from our analytical criterion. The dots are results from the SPH models for the face-on configuration, compared to the predictions, adopting a value of $A=0.5$ (solid curve). The open circles are results in edge-on orientation, and the dashed curves are the predictions with an adopted value $A=0.12$. The dotted curves come from the fitting formula Eq. (2).

i.e. if the speed $v_{\text {after }}$ exceeds the local escape speed. The stripping radius would then be defined as the radius where $v_{\text {after }}=v_{\text {esc }}$ and the stripped mass fraction as the mass exterior to that radius:

$M_{\text {strip }}=\int_{r_{\text {strip }}}^{r_{\text {out }}} 2 \pi \Sigma_{\mathrm{ISM}}(r) r \mathrm{~d} r / M\left(r_{\text {out }}\right)$,

where $r_{\text {out }}$ is the truncation radius of the disk. For an exponential $\operatorname{disk} \Sigma_{\mathrm{ISM}}(r)=\Sigma_{0} \exp \left(-r / a_{d}\right)$ with $M_{\mathrm{ISM}}=2 \pi \Sigma_{0} a_{d}^{2}$ we have

$M_{\text {strip }}=\frac{\left(a_{\mathrm{d}}+r_{\text {out }}\right) \mathrm{e}^{-r_{\text {out }} / a_{\mathrm{d}}}-\left(a_{\mathrm{d}}+r_{\text {strip }}\right) \mathrm{e}^{-r_{\text {strip }} / a_{\mathrm{d}}}}{\left(a_{\mathrm{d}}+r_{\text {out }}\right) \mathrm{e}^{-r_{\text {out }} / a_{\mathrm{d}}}-a_{\mathrm{d}}}$.

On the other hand, the escape velocity as a function of galactocentric radius is the sum of the contributions from all components, i.e. bulge, disk, and dark halo. At the stripping radius the escape velocity equals the speed $v_{\text {after }}$, which is a function of the ICM column density which we shall now take as the independent variable. Hence we can compute the stripping radius as a function of ICM column density, and with that we get the stripped mass fraction. This is easily evaluated numerically by computing for each galactocentric radius the mass fraction outside of that radius, and on the other hand the quantity

$\Sigma_{\mathrm{ICM}}=v_{\mathrm{esc}} \frac{\Sigma_{\mathrm{ISM}}(r)}{\langle v\rangle_{\rho_{\mathrm{ICM}}}}=A \sqrt{-2 \Phi(r)} \frac{\Sigma_{\mathrm{ISM}}(r)}{\langle v\rangle_{\rho_{\mathrm{ICM}}}}$.

Figure 14 depicts the relation between $M_{\text {strip }}$ and $\Sigma_{\text {ICM }}$ for our standard model. The above equation is able to match the SPH results very closely, if we adopt $A=0.5$. In this diagram, our fit formula to the SPH results (Eq. (2)) gives a function very similar in character to the one derived from our analytical criterion. The choice of the outer disk radius influences the shape of the curve in Fig. 14; we find that a value of $20 \mathrm{kpc}$ gives a better match than the value of $16 \mathrm{kpc}$ assumed for the SPH models. This detail is not incorporated in the fit formula, whose results therefore match the SPH results at low stripping less well.

Although the SPH results from the edge-on case do exhibit a larger scatter than the face-on data, it is quite remarkable that they can still be matched by the analytical prediction for the faceon case, if one simply increases the necessary ICM column density. This means that the edge-on models can well be considered 


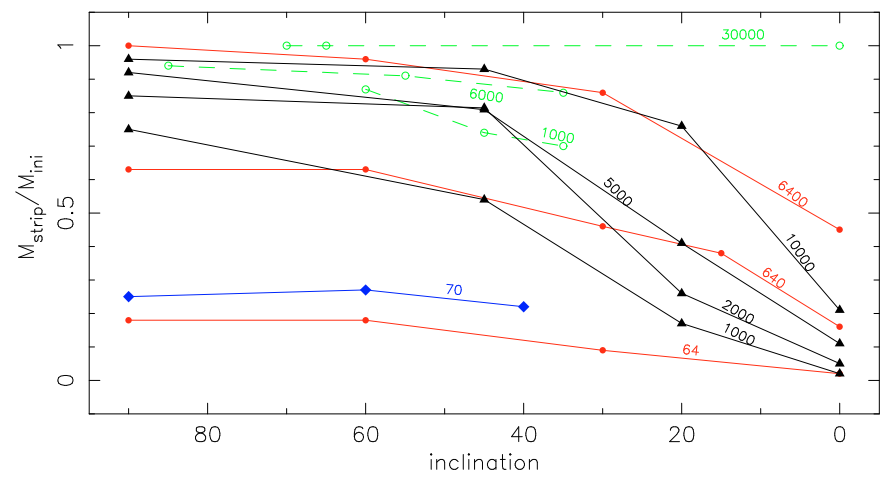

Fig. 15. Stripped mass fraction as a function of the disk inclination, from Vollmer et al. (2001) - black, Roediger \& Brüggen (2006) red, Roediger \& Brüggen (2007) - green, and Schulz \& Struck (2001) - blue. Labels give values of the peak ram pressure, in units of $\mathrm{cm}^{-3}\left(\mathrm{~km} \mathrm{~s}^{-1}\right)^{2}$. For Roediger \& Brüggen (2007) the actual values of the inclination are given for the instant of pericenter passage.

as having a lower stripping efficiency, with a reduction factor of about 5. This factor may be regarded as depending only on the tilt angle $i$, as

$A(i)=0.12+0.38 \sin i$.

A sine-type dependence could be understandable as a geometrical projection effect, in that the edge-on galaxy presents a smaller cross section to the ICM. If it were such a pure effect of the effective cross sections, one should expect for our standard model a ratio of the areas of $4 / 0.25=16$, from the disk radial scale being $4 \mathrm{kpc}$ and its height being $0.25 \mathrm{kpc}$. For the moment, we prefer to take our analytical formula as a convenient expression which encapsulates essential features of the problem rather than as giving a complete description.

\section{Discussion}

We compare our results to the findings of previous numerical studies which treated inclined ram pressure stripping. These investigations differ in their numerical treatment of hydrodynamics, the model for the galaxy, and the way the ICM gas flows around the galaxy.

\subsection{General trends}

The fraction of the ISM mass that is stripped in the simulations of Vollmer et al. (2001), Roediger \& Brüggen (2006), Roediger \& Brüggen (2007), and Schulz \& Struck (2001) is plotted in Fig. 15 as a function of inclination angle. The curves join runs with different disk inclinations, and are labeled by the maximum ram pressure. It must be stressed that for different curves not only does the maximum value of the ram pressure vary, but also its duration. These general features are apparent in Fig. 15:

- for inclinations larger than about $50^{\circ}$ there is very little dependence of the stripped fraction;

- towards the edge-on orientation, on the contrary, the amount of stripping drops strongly;

- the curves flatten for higher peak ram pressures.

Our results show the same features (cf. Fig. 7). A closer comparison shows that the near edge-on models by Vollmer et al. (2001) are substantially more strongly stripped than ours for the same peak ram pressure. However, since their Fig. 7 suggests that the

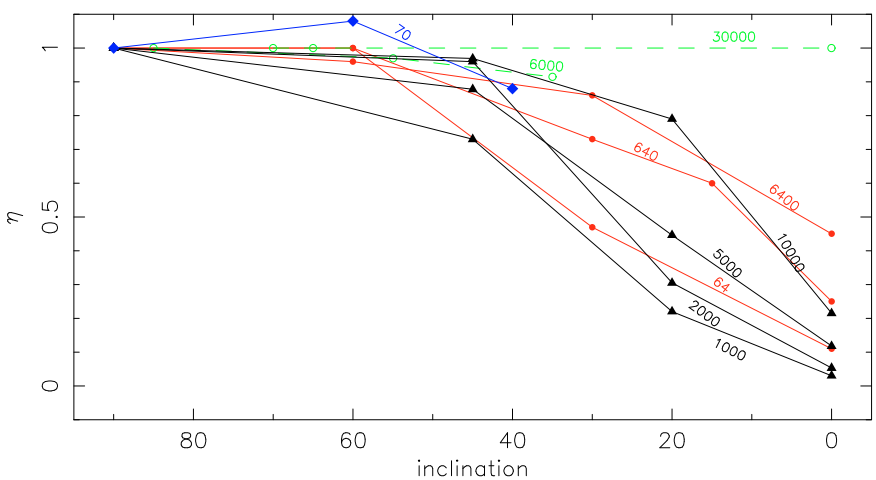

Fig. 16. Stripping efficiency with respect to inclination as a function of the angle for the same results as in Fig. 15.

radial scale-length of the gas disk is longer than in our model, one should expect that due to the higher ISM surface density they should be stripped less severely. This suggests that the shielding mechanism used by Vollmer et al. (2001) is overly effective in near edge-on orientations and thus overestimates the stripping, at least in comparison to the full hydrodynamic treatment of Roediger \& Brüggen (2006) and our SPH approach.

For the same values of the stripped mass fraction, the simulations by Roediger \& Brüggen (2006) require a substantially lower value of the peak ram pressure than our models or those by Vollmer et al. (2001), because Roediger \& Brüggen (2006) consider the flow of the ICM to be constant in time, hence much longer than in the other models.

The stripping efficiency with respect to inclination $(\eta)$ measures the relative stripping potential of individual inclinations with respect to the face-on case. It might thus serve as a good instrument for comparing results from different numerical codes. From Fig. 16 it is obvious that the results of different authors give very similar dependences on the inclination angle. In spite of the basic difference in the absolute values for the maximum ram pressure, the results of both Roediger \& Brüggen (2006) and Vollmer et al. (2001) show that higher ram pressures result in a lower dependence of the stripping on inclination angle, i.e. making the inclined stripping as effective as the face-on case. Thus, the features of the inclination dependence, which our present study shows in a systematic way, has already been seen in earlier studies.

\subsection{Vollmer's fitting formula}

Vollmer et al. (2001) derived from their simulation results a formula which relates the fraction of the final and initial total gas mass to the peak ram pressure and inclination angle (their Eq. (21)): $M_{\text {ini }} / M_{\text {fin }}=0.25 p_{\text {ram }}^{\max } \sin ^{2}\left[0.9\left(i+10^{\circ}\right)\right]+0.84$, where $p_{\mathrm{ram}}^{\max }$ is normalized by $p_{0}=100 \mathrm{~cm}^{-3}\left(\mathrm{~km} \mathrm{~s}^{-1}\right)^{2}$. Using this fitting formula we reproduce Vollmer's Fig. 17 in Fig. 17: as a function of angle we plot the stripping efficiency with respect to inclination $\eta$. It shows that although the fitting function does not match the simulations perfectly (and even gives for low ram pressures negative values of $\eta$ ) the general trend corresponds well to the numerical results, and to our findings as well: that for high ram pressures the efficiency of stripping is high and less dependent on the tilt angle than for low ram pressure values. 


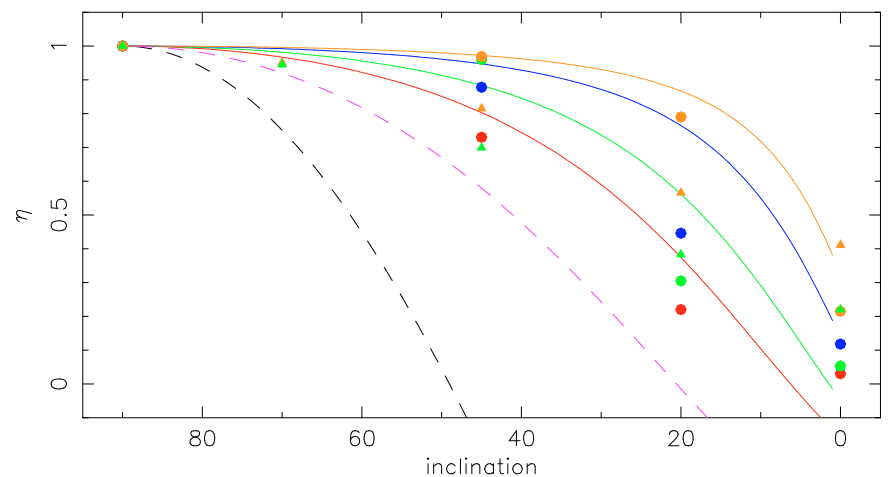

Fig. 17. Curves of stripping efficiency with respect to inclination $\eta$ as a function of tilt angle as calculated from the fitting formula of Vollmer et al. (2001) for various values of the peak ram pressure: 100, $300,1000,2000,5000$ and $10000 \mathrm{~cm}^{-3}\left(\mathrm{~km} \mathrm{~s}^{-1}\right)^{2}$ (from left to right). Results of Vollmer's simulations (dots) correspond to the solid curves. Results of our $R 4 \rho 0$ and $R 4 \rho 1$ runs with pressure peaks of $\sim 2700$ and $11000 \mathrm{~cm}^{-3}\left(\mathrm{~km} \mathrm{~s}^{-1}\right)^{2}$ are added (triangles).

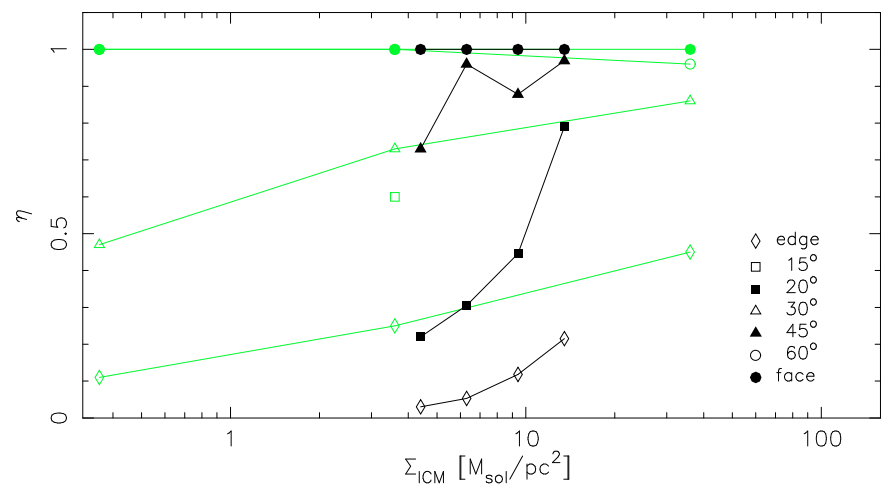

Fig. 18. Stripping efficiency with respect to inclination $\eta$ as a function of the column density of the encountered ICM for simulations of Roediger \& Brüggen (2006) (green) and Vollmer et al. (2001) (black).

\subsection{Dependence on ICM column density}

To look for the dependence of the stripping results on $\Sigma_{\text {ICM }}$ in the previous simulations, we estimate these values from the parameters of their runs. In Roediger \& Brüggen (2006), a constant flow of ICM with three values of density $(0.01,0.1$, and $1 \times 10^{-26} \mathrm{~g} \mathrm{~cm}^{-3}$ ) provokes a wind at $800 \mathrm{~km} \mathrm{~s}^{-1}$ lasting for 1 Myr. This yields values of the ICM column density of 0.4 , 4 , and $36 M_{\odot} \mathrm{pc}^{-2}$ for their weak, medium, and strong pressure models, respectively. Vollmer et al. (2001) approximate ram pressure profiles with Lorentz functions, whose duration is determined by the condition that $500 \mathrm{Myr}$ before the peak, a constant pressure of $50 \mathrm{~cm}^{-3}\left(\mathrm{~km} \mathrm{~s}^{-1}\right)^{2}$ is reached. For their four peak ram pressures of 1000, 2000, 5000, and $10000 \mathrm{~cm}^{-3}\left(\mathrm{~km} \mathrm{~s}^{-1}\right)^{2}$ we derive widths $t_{\mathrm{HW}}=115,80,50$, and $35 \mathrm{kpc}$, respectively, which yield values of $\Sigma_{\mathrm{ICM}}: 4.4,6.2,9.4$, and $13.5 M_{\odot} \mathrm{pc}^{-2}$.

Figure 18 compares the $\eta\left(\Sigma_{\text {ICM }}\right)$ dependence for the results of Vollmer et al. (2001) and Roediger \& Brüggen (2006). We do not include the results of Roediger \& Brüggen (2007), as the tilt angle changes along the galaxy's orbit. The results of both Vollmer et al. (2001) and Roediger \& Brüggen (2006) show the same trend as that revealed in our simulations: with increasing $\Sigma_{\text {ICM }}$ the inclination efficiency grows. It is worth noting that the increase is much steeper in the case of Vollmer et al. (2001), in comparison to Roediger \& Brüggen (2006) and our own results. Since the parameters of the model galaxies are rather similar and the stripping efficiency $\eta$ measures the relative potential of inclined stripping with respect to the face-on case, it could well be that these differences are due to the different ways the hydrodynamics is treated.

\subsection{Limitations of strictly radial orbits}

To separate the effects of the disk tilt angle on the stripping results we have focused in this paper on galaxies on strictly radial orbits. Then the tilt angle stays constant along the orbit. In more realistic orbits however, the disk inclination usually changes with time. Thus, almost all galaxies are likely to experience once along their orbit an efficient (close-to) face-on ram pressure phase. Its duration and strength is then determined by the parameters of the orbit. As the results of our simulations have shown, the tilt-variance might be important for situations with compact ICM distributions where the face-on stripping really is more efficient than the tilted ones. In extended clusters, however, the dependence on the disk inclination vanishes as the stripping tends to saturate.

Concerning the stripping potential of individual models, note that the strictly radial orbits used in our calculations may be considered as being equivalent to slightly elliptical ones: The ram pressure profile along a strictly radial orbit through a given ICM distribution may well be the same as the ram pressure profile along an elliptical orbit, however through a modified ICM distribution that is more centrally peaked (higher) and less extended (more compact). In other words, on an elliptical orbit the galaxy passes through the slope of the ICM density peak.

\section{Conclusion}

We have studied in detail the influence of disk tilt angle on the ram pressure stripping efficiency in galaxy clusters. A grid of $\mathrm{N}$-body/SPH numerical simulations, varying the density of the ICM, the peak ram-pressure, its duration, and the orientation of the galaxy was performed. We summarize below the main results of the simulations:

- the stripping amount is lower for galaxies moving edge-on with respect to the ICM wind, in agreement with previous simulations;

- apart from the total stripped amount, there is no radical difference in the duration of the stripping process of variously tilted galaxies;

- due to conservation of angular momentum, ram pressure shifts the ISM in near-edge-on orientations to smaller distances behind the galaxy than in the face-on case;

- the dependence of the stripping amount on the disk tilt angle vanishes for large clusters, where the amount of the encountered ICM $\left(\Sigma_{\mathrm{ICM}}\right)$ is large;

- on the other hand, for galaxies crossing small ICM overdensities or debris structures left over in the cluster from recent stripping events, the dependence on the tilt angle is more pronounced;

- we emphasize the role of the amount of the encountered ICM to the ram pressure stripping results;

- the stripping efficiency with respect to inclination $\eta$ (i.e. the ratio of the stripped mass fraction relative to the face-on case) depends strongly on the disk tilt angle for small ram pressure peaks but again vanishes for large values of $\Sigma_{\text {ICM }}$ when stripping saturates; 
- for non-face-on orientations, the sense of rotation plays a role: contrary to expectations, it is not the side with highest ram pressure which is more stripped;

- we propose a fitting formula (Eq. (2)), which should now be tested against observations, deriving the ICM density from $\mathrm{X}$-ray imaging of clusters, and the gas stripping from $\mathrm{HI}$ observations.

By comparison with a simple analytical stripping condition (Jáchym et al. 2007), the total momentum imparted by the encountered ICM on the galaxy gas appears to be the main determinant of the stripping result. Contrary to the GG72 criterion or the fitting formula of Vollmer et al. (2001) who assign the key role to the peak value of the ram pressure, we stress the importance of the dependence on the amount of the encountered ICM, together with the disk inclination angle:

$M_{\text {strip }} / M_{\text {init }} \sim \Sigma_{\mathrm{ICM}}\left(a+b \sin ^{\alpha} i\right)$.

The values of $a, b$, and $\alpha$ depend on the ISM distribution in the unperturbed disk. We use such a dependence to fit the outcome of our numerical simulations - the stripped mass fraction and the stripping radius. The analytical stripping condition based on the momentum balance yields similar formulae.

Using simple test particle models taking schematically into account the "shadowing" of the gas disk by the material occurring upstream, we can roughly obtain the main features of the stripping. The imparted momentum $\langle v\rangle_{\rho_{\mathrm{ICM}}} \Sigma_{\mathrm{ICM}}$ proves to be the key parameter of the ICM-ISM interaction. Such particle models however fail to reflect the true dependence of the stripping efficiency on ICM density and inclination revealed by the full hydrodynamics.

Acknowledgements. The authors gratefully acknowledge support by the Institutional Research Plan AV0Z10030501 of the Academy of Sciences of the Czech Republic, by the project 205/08/P556 of the Grant Agency of the Czech Republic, and by the Center for Theoretical Astrophysics (LC06014). We thank Volker Springel for valuable advice. The simulations have been carried out on the Ondřejov Cluster for Astrophysical Simulations (OCAS, Czech Republic) and the VIRGO computational cluster in Prague. We thank Jim Dale for English revision of the text. We would like to thank the referee for helping us to improve this paper considerably.

\section{References}

Abadi, M. G., Moore, B., \& Bower, R. G. 1999, MNRAS, 308, 947 Agertz, O., Moore, B., Stadel, J., et al. 2007, MNRAS, 380, 963 Cavaliere, A., \& Fusco-Femiano, R. 1976, A\&A, 49, 137

Chung, A., van Gorkom, J. H., Kenney, J. D. P., \& Vollmer, B. 2007, ApJ, 659, L115

Crowl, H. H., Kenney, J. D. P., van Gorkom, J. H., \& Vollmer, B. 2005, AJ, 130, 65

Farouki, R., \& Shapiro, S. L. 1980, ApJ, 241, 928

Gingold, R. A., \& Monaghan, J. J. 1977, MNRAS, 181, 375

Gunn, J. E., \& Gott, J. R. I. 1972, ApJ, 176, 1

Hidaka, M., \& Sofue, Y. 2002, PASJ, 54, 33

Jáchym, P., Palouš, J., Köppen, J., \& Combes, F. 2007, A\&A, 472, 5

Kapferer, W., Kronberger, T., Ferrari, C., Riser, T., \& Schindler, S. 2008, MNRAS, 389, 1405

Kawata, D., Okamoto, T., Cen, R., \& Gibson, B. K. 2009, ArXiv e-prints

Kitsionas, S., \& Whitworth, A. P. 2007, MNRAS, 378, 507

Kronberger, T., Kapferer, W., Ferrari, C., Unterguggenberger, S., \& Schindler, S. 2008, A\&A, 481, 337

Lucy, L. B. 1977, AJ, 82, 1013

Marcolini, A., Brighenti, F., \& D'Ercole, A. 2003, MNRAS, 345, 1329

Okamoto, T., Jenkins, A., Eke, V. R., Quilis, V., \& Frenk, C. S. 2003, MNRAS, 345,429

Phookun, B., \& Mundy, L. G. 1995, ApJ, 453, 154

Quilis, V., Moore, B., \& Bower, R. 2000, Science, 288, 1617

Roediger, E., \& Brüggen, M. 2006, MNRAS, 369, 567

Roediger, E., \& Brüggen, M. 2007, MNRAS, 380, 1399

Roediger, E., \& Brüggen, M. 2008, MNRAS, 388, 465

Schindler, S., Binggeli, B., \& Böhringer, H. 1999, A\&A, 343, 420

Schulz, S., \& Struck, C. 2001, MNRAS, 328, 185

Springel, V. 2009, ArXiv e-prints

Springel, V., Yoshida, N., \& White, S. D. M. 2001, New Astron., 6, 79

Tonnesen, S., \& Bryan, G. L. 2008, ApJ, 684, L9

Tonnesen, S., \& Bryan, G. L. 2009, ApJ, 694, 789

Vollmer, B., Cayatte, V., Balkowski, C., \& Duschl, W. J. 2001, ApJ, 561, 708

Vollmer, B., Soida, M., Chung, A., et al. 2009, A\&A, 496, 669 